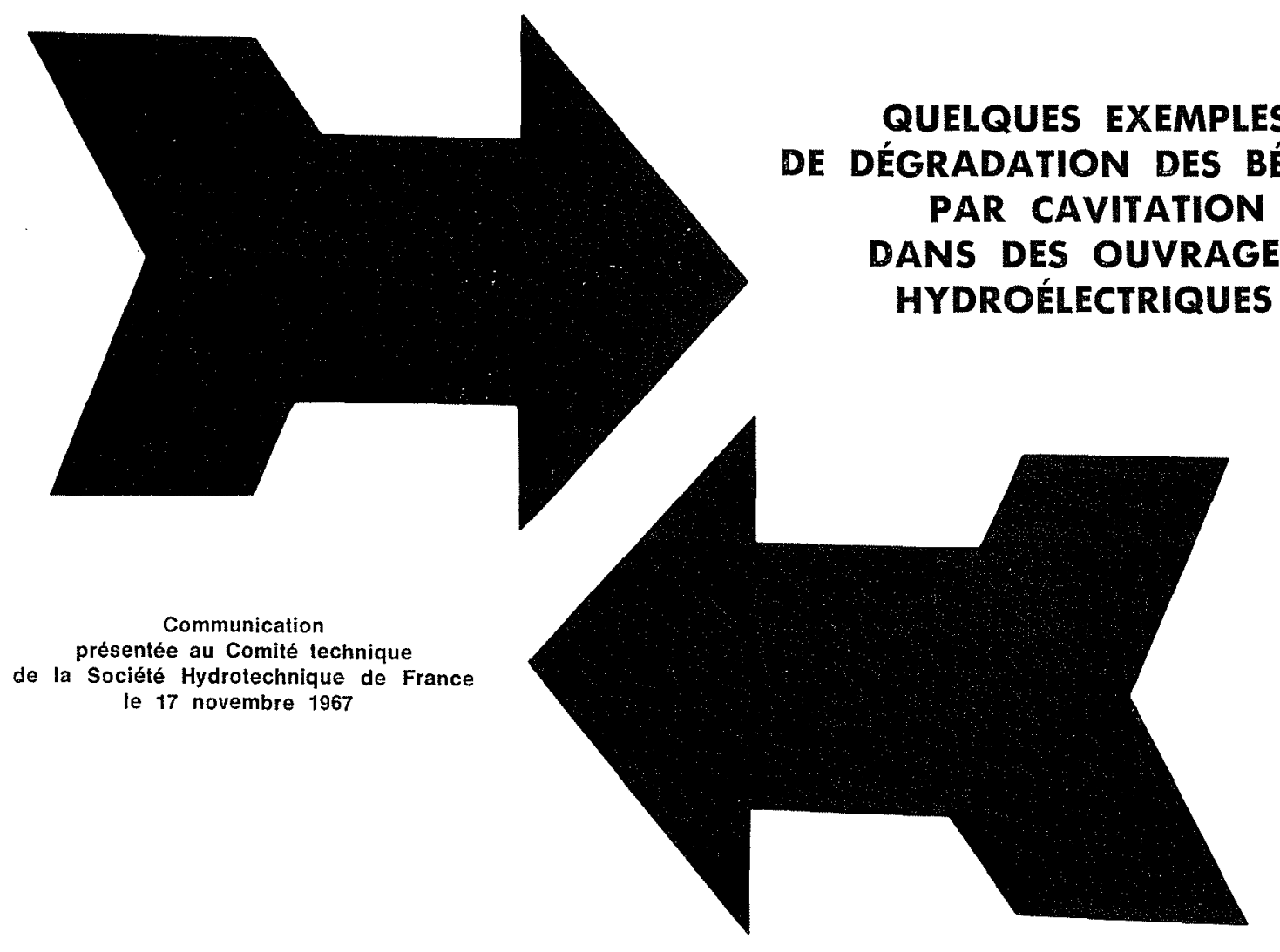

\title{
DE DÉGRADATION DES BÉTONS PAR CAVITATION DANS DES OUVRAGES HYDROÉLECTRIQUES
}

PAR J. DESTENAY * ET J. BERNARD * *

A la demande de la Société Hydrotechnique de France -... Section « Machines »-.- le Service de la Production Hydraulique d'Electricité de France : effectué, en 1965, une enquête sur les dégradations provoquées par la cavitation dans les ouvrages de génie civil soumis à l'action de l'eau.

De l'observation des formes constructives et des conditions d'écoulement correspondant aux diver's cas recensés, il semble possible de lirer les quelques enseignements suivants :

- les dégradations sont relativement peu nombreuses;

- les érosions sont habituellement faibles et celles de quelque importance ont été observées, le plus souvent, après des durées de fonctionnement très supérieures à celles de l'exploitation normale;

- les cas rencontrés semblent pouvoir entrer, schématiquement, dans deux catégories d'écoulement :

a) écoulement de vitesse modérée $(10-20 \mathrm{~m} / \mathrm{s})$ à forte turbulence;

b) écoulement de vitesse élevée (au-delà de $30 \mathrm{~m} / \mathrm{s}$ ) à faible turbulence.

Les résultats de l'enquête seront donc exposès,

\footnotetext{
* Ingénieur-Conseil auprès d'E.D.F.

* Ingénieur au Service de la Production Hydraulique d'E.D.F'.
}

dans le présent rapport, suivant les quatre paragraphes ci-apres :

I. - Nombre (et proportion) d'ouvrages ayant subi des dommages par fait de cavitation;

II. - Données concernant les durées de fonctionnement des principaux ouvrages détériorés;

III. - Exemples de dégradations pour des ouvrages où la vitesse moyenne d'écoulement est modérée (catégorie a);

IV. - Exemples de dégradations pour des ouvrages où la vitesse moyenne d'écoulement est élevée (catégorie $b$ ).

\section{Nombre d'ouvrages ayant subi} des dommages

Le Service de la Production Hydraulique exploitait, en 1965, environ 400 aménagements hydroélectriques appartenant, soit à l'Electricité de France, soit à la Compagnie Nationale du Rhòne.

Il s'agit d'aménagements spécifiquement industriels, donc conçus et traités comme tels. En conséquence, dans la grande majorité des cas, les ouvrages ont été étudiés pour éviter, autant que possible, les dégradations, en particulier celles dues à la cavitation et, le plus souvent, le but a été parfaitement atteint.

Le nombre de cas intéressant le présent rapport s'en trouve considérablement réduit et encore pour 


\section{J. DESTENAY et J. BERNARD}

les ouvrages où le projeteur a été contraint d'accepter le risque de cavitation, il s'est, bien entendu, efforcé de le minimiser. De ce fait, certaines dégradations sont souvent assez faibles ou d'origine douteuse et il n'est pas possible de les repertorier avec suffisamment de certitude dans ce rapport. Enfin, les nécessités de l'exploitation n'ont pas toujours permis de procéder à toutes les investigations nécessaires.

Dans ces conditions, on n'a observé que quelques cas, une vingtaine au total, inattendus ou correspondant à des fonctionnements exceptionnellement prolongés.

Une population aussi restreinte et aussi spéciale ne permet pas d'accorder aux tableaux que nous présentons - pour faciliter l'exposé - une quelconque valeur statistique. D'ailleurs, la présente enquête avait bien été prévue pour corroborer, par des exemples industriels, des résultats d'ordre plus ou moins théorique et pour fournir des exemples " grandeur nature » complétant ceux obtenus, en laboratoire, par des séries d'essais systématiques.

A titre indicatif, nous donnons ci-dessous la répartition des cas de dégradations, par cavitation, dans les divers types d'ouvrages.

\begin{tabular}{|c|c|}
\hline TYPe n'ovyrage & $\begin{array}{l}\text { NOMBRE } \\
\text { DE CAS } \\
\text { OBSERVÉS }\end{array}$ \\
\hline Evacuateur de surface............. & 1 \\
\hline Evacuateur de demi-fond........... & 1 \\
\hline 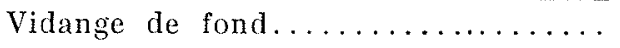 & 2 \\
\hline 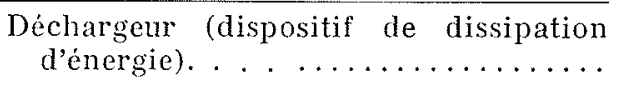 & 6 \\
\hline Aspirateur de turbine.............. & 3 \\
\hline Zone aval rainure de vanne......... & 4 \\
\hline Ouvrages divers. . . .............. & 4 \\
\hline Total. & 21 \\
\hline
\end{tabular}

Il peut sembler intèressant de rapprocher, de certains de ces chiffres, le nombre d'ouvrages, de même type, en service à l'époque de l'enquête. Il convient cependant d'être très prudent dans toute tentative d'analyse, car il n'existe pas deux ouvrages absolument identiques (forme, nature des ma-

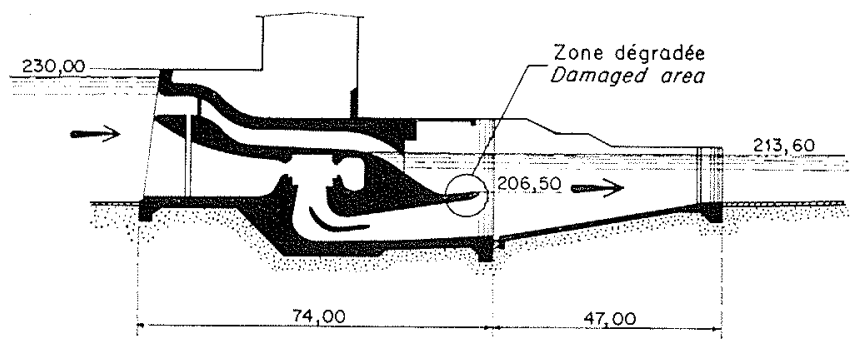

1/Ottmarsheim : ensemble du déchargeur.

Ottmarsheim : general arrangement of relief works. tériaux, fini de surface, etc.) ayant fonctionné exactement dans les mêmes conditions ou ayant eu des durées de service comparables pour des régimes d'écoulement voisins.

\begin{tabular}{|c|c|c|}
\hline TYPE D'OUVRAGE & $\begin{array}{l}\text { NOMBRE } \\
\text { DE TYPE } \\
\text { D'OUVRAGES } \\
\text { COMPARABLE } \\
\text { EN SERVICE } \\
\text { EN } 1965\end{array}$ & $\begin{array}{c}\text { NOMBRE } \\
\text { DE CAS } \\
\text { D'ÉROSION } \\
\text { PAR } \\
\text { CAVITATION } \\
\text { OBSERVÉS } \\
\text { EN } 1965\end{array}$ \\
\hline $\begin{array}{l}\text { Evacuateur de surface: } \\
\text { Type «saut de ski ».... } \\
\text { En galerie souterraine... }\end{array}$ & $\begin{array}{l}18 \\
13\end{array}$ & $\overline{2}$ \\
\hline $\begin{array}{l}\text { Déchargeur: } \\
\text { Basse chute }(10 \text { à } 25 \mathrm{~m}) \text {. } \\
\text { Chute moyenne }(25 \mathrm{~m} \text { à } \\
70 \mathrm{~m}) . \ldots \ldots \ldots\end{array}$ & 10 & 5 \\
\hline
\end{tabular}

On voit donc que, pour les ouvrages en service en 1965 et avec les durées de fonctionnement atteintes à celte époque, les cas de dégradation par cavitation restent relativement rares sauf, peutêtre, pour les déchargeurs (50\% environ).

\section{Données concernant les durées de fonctionnement}

Certaines des dégradations observées se sont produites sur des ouvrages ayant eu des durées de fonctionnement exceptionnelles au moment de leur mise en service. C'est le cas, notamment, de quelques déchargeurs et de la vidange de fond $n^{\circ} 1$ de Serre-Ponçon.

On peut citer, par exemple, les fonctionnements suivants :

- à Fessenheim, pour permettre le fonctionnement des usines amont et à la navigation d'emprunter le canal pendant le montage des groupes à l'usine, il est passé, entre septembre 1956 et l'été 1957, 8 milliards de mètres cubes d'eau par chacun des deux pertuis du déchargeur. L'énergie dissipée pendant cette période correspond à $600 \mathrm{GWh}$ environ;

- à Saint-Estève-Janson, au cours de l'été 1963 , le volume d'eau évacué par le déchargeux pendant l'achèvement du montage des groupes a été d'un milliard de mètres cubes;

- a Serre-Poncon, la vidange de fond $\mathbf{n}^{\circ} 1$ a évacué, entre novembre 1959 et juillet $1960,1,56$ milliard de mètres cubes sous des charges variant entre 60 et $100 \mathrm{~m}$.

Par contre, pour d'autres ouvrages, on a constaté des dégradations pour des durées de fonctionnement beaucoup plus réduites.

Par exemple, sur le déchargeur de Châteauneufdu-Rhône, les premières constatations d'èrosions sur les redans des chambres de dissipation d'énergie remontent à une époque où les ouvrages avaient fonctionné $20 \mathrm{~h}$ en déchargeur et $250 \mathrm{~h}$ pour les 
vidanges de l'écluse (l'aqueduc de vidange de l'écluse débouche à l'amont de la chambre de dissipation d'énergie).

Sur les quatre pertuis du barrage de Rivières, sur le Tarn, équipés de vannes wagons, on a fait les observations suivantes sur les bajoyers des piles, immédiatement en aval des rainures des vannes.

\begin{tabular}{|c|c|c|}
\hline $\begin{array}{l}\text { Pentuis } \\
\mathrm{N}^{\circ}\end{array}$ & $\begin{array}{l}\text { DURÉE } \\
\text { CUMULÉE DE } \\
\text { FONCTION- } \\
\text { NEMENT } \\
\text { (h) }\end{array}$ & EROSIONS OBSERVÉES \\
\hline 1 & 250 & $\begin{array}{l}\text { Rive droite : profon- } \\
\text { deur maximale. . . } 6 \mathrm{~cm} \\
\text { Rive gauche : profon- } \\
\text { deur maximale. . } 10 \mathrm{~cm}\end{array}$ \\
\hline 2 & 630 & $\begin{array}{l}\text { Profondeur maximale. } 22 \mathrm{~cm} \\
\text { Arrachement a la crue } \\
\text { de } 1965 \text { de blindages } \\
\text { mis en } 1964 .\end{array}$ \\
\hline 3 & 990 & $\begin{array}{l}\text { Rive droite : profon- } \\
\text { deur maximale. . . } 10 \mathrm{~cm} \\
\text { Rive ganche : profon- } \\
\text { deur maximale. . . } 7 \mathrm{~cm}\end{array}$ \\
\hline 4 & 50 & $\begin{array}{c}\text { Premières traces de } \\
\text { «martèlement». }\end{array}$ \\
\hline
\end{tabular}

Il est possible de penser qu'une certaine « durée d'incubation » est nécessaire avant l'apparition des premières dégradations et qu'elle peut varier, dans d'assez grandes limites, pour des ouvrages de même type.

\section{Exemples de dégradations par cavitation au sein d'écoulements à vitesse moyenne modérée mais à forte turbulence}

\section{Dégradations observées dans des déchargeurs.}

Ce type d'écoulement se rencontre, en particulier, dans les zones de dissipation d'énergie des déchargeurs. Des dégradations ont été observées sur des déchargeurs de basse chute, notamment à Ottmarsheim (chute brute : $16 \mathrm{~m}$ ), Fessenheim $(15 \mathrm{~m})$, Vogelgrün $(12,50 \mathrm{~m})$, Bollène $(23 \mathrm{~m})$ et Châteauneuf $(18,30 \mathrm{~m})$ et sur un ouvrage de chute moyenne : Saint-Estève-Janson $(65 \mathrm{~m})$.

A Ottmarsheim, six pertuis de décharge sont associés à chaque groupe. Ils se terminent par une doucine située au-dessus du débouché des aspirateurs (fig. 1).

A la mise en service, en 1952, après six mois de fonctionnement ininterrompu, les extrémités des doucines des déchargeurs ont dû être rebétonnées, les armatures ayant été mises à nu.

A Fessenheim, au lieu de répartir les déchargeurs sur toute la longueur de l'usine, comme à Ottmarsheim, on a accolé, au centre de l'usine, deux déchargeurs capables chacun de $600 \mathrm{~m}^{3} / \mathrm{s}$ (fig. 2).

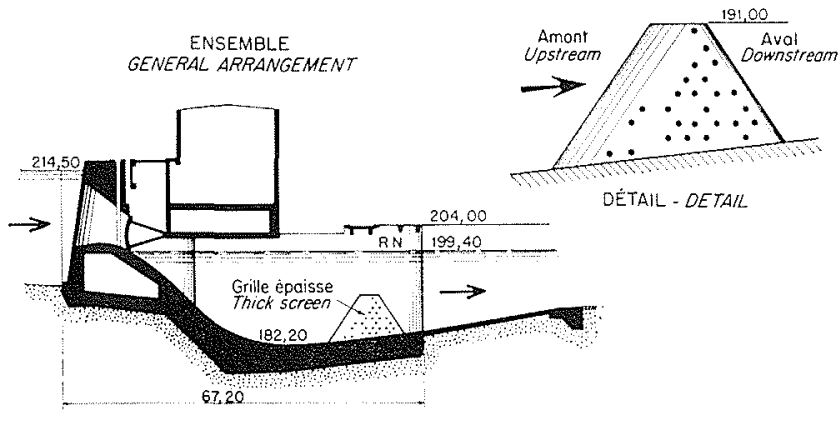

2/ Fessenheim : ensemble du dèchargenr. Détail de la grille. Fessenheim : general arrangement of relief works. Screen details.

Pour amortir la lâchure d'un débit voisin de $1200 \mathrm{~m}^{3} / \mathrm{s}$ à une vilesse de 10 à $12 \mathrm{~m} / \mathrm{s}$, on a installé, au point bas des passes, une grille épaisse constituée de 26 tubes en acier, de $32 \mathrm{~cm}$ de diamètre. Ceux-ci sont remplis de béton pour accroître leur inertie et sont ancrés de $47 \mathrm{~cm}$ à leurs extrémités. Le point haut de la grille se trouve à 8 ou $9 \mathrm{~m}$ en dessous du niveau moyen du bief aval.

Après le fonctionnement exceptionnel indiqué plus haut, une visite des deux déchargeurs, par les hommes-grenouilles, eut lieu en 1958. Ils constatèrent la disparition du tube le plus proche du radier sur toute la largeur des deux déchargeurs et des dégradations du revêtement des bajoyers des passes et des pilettes intermédiaires qui divisent en trois pertuis l'extrémité aval de chaque déchargeur (photo I).

Après batardage des passes, on s'apercut que le revêtement d'usure des bajoyers et des pilettes, constitué d'une couche de gunite de $25 \mathrm{~mm}$ d'épaisseur, présentait des érosions caractéristiques de cavitation dans le sillage des tubes. Les zones dégradées atteignaient une longueur de $50 \mathrm{~cm}$ et une profondeur de 30 à $40 \mathrm{~mm}$. Le revètement d'usure a été reconstitué avec un mortier de ciment contenant un durcisseur.

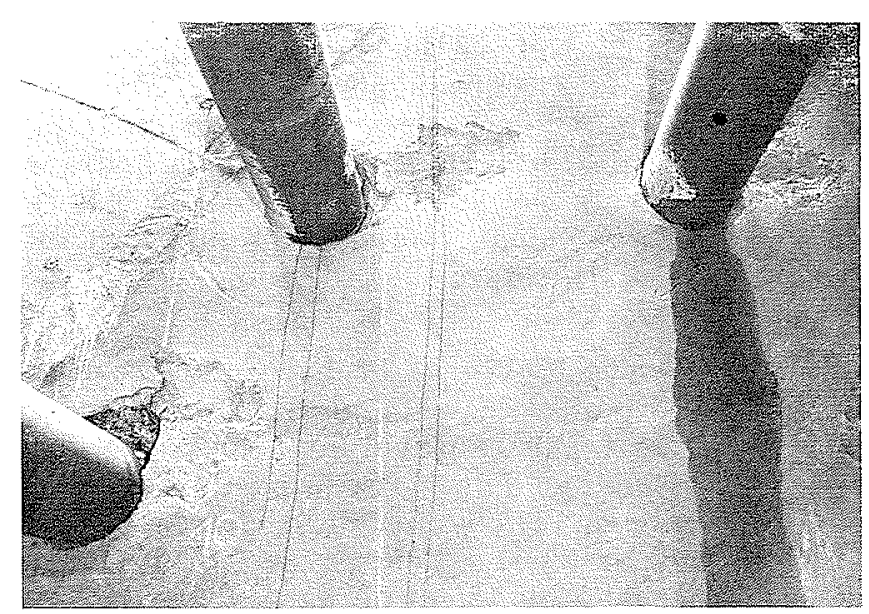

Photo I/

(Photo 7.-P. Sclawarte, Mulhouse.)

Fessenheim : érosions dans le sillage des tubes.

Fessenheim: erosion doun the tube wakes. 
A Vogelgrün, le dispositif d'amortissement est identique, dans son principe, à celui de Fessenheim; certaines modifications de détail ont cependant été apportées à la suite de l'expérience de Fessenheim :

-.-- gainage des tubes avec du caoutchouc, sur $10 \mathrm{~cm}$ de longueur, au niveau de leurs ancrages, pour éviter la transmission des vibrations au béton;

- longueur d'ancrage portée de 50 à $90 \mathrm{~cm}$;

- remplissage des trois tubes inférieurs par du mortier bitumineux.

Une visite du déchargeur eut lieu a l'automne 1959 .

Sur le déchargeur rive droite, apris $4000 \mathrm{~h}$ de fonctionnement, on releva des zones d'érosion du béton situées dans le sillage des tubes et d'une profondeur variant entre 2 et $20 \mathrm{~cm}$. Ces zones concernaient principalement les tubes amont.

Chaque pilette était, en outre, fissurée sur ses faces amont et aval.

Sur le dechargeur rive gauche, apres $2300 \mathrm{~h}$ de fonctionnement, les zones d'érosion du béton étaient moins nombreuses qu'en rive droite mais de même importance unitaire. Les fissures sur les pilettes étaient également moins longues.

Les dégradations ont été réparées avec un mortier de ciment additionné de $20 \%$ d'oxydociment.

A Châteauneuf-du-Rhône, le déchargeur est constitué de deux pertuis semblables, alimentés chacun par une vanne-segment de fond et un clapet de surface. Chaque pertuis est capable d'évacuer $570 \mathrm{~m}^{3} / \mathrm{s}$, dont $480 \mathrm{~m}^{3} / \mathrm{s}$ par la vanne de fond. La dissipation d'énergie s'effectue dans une chambre de $35 \mathrm{~m}$ de longueur et de $8 \mathrm{~m}$ de largeur, munie de 6 « dents de fond » dont les formes et les emplacements ont été étudiés sur modèle réduit. Le système de dents évite, en outre, que le déchargeur en fonctionnement ne joue le rôle d'éjecteur pour l'aqueduc de vidange de l'écluse qui débouche à proximité de la vanne de fond (fig. 3 ).

Les premières constatations de dégradations notables sur les dents de dissipation d'énergie ont été effectuées en décembre 1958 , après $270 \mathrm{~h}$ de fonctionnement, soit en déchargeur $(20 \mathrm{~h})$, soit pour la vidange de l'écluse $(250 \mathrm{~h})$.

Les zones de béton érodées se situaient notamment à l'aval des arêtes ou au niveau de l'enracinement des dents sur le radier (photo II). A l'origine, les dents étaient blindées uniquement sur certaines faces. Quelques tôles de blindage ont été également arrachées.

A Saint-Estève-Janson, le déchargeur est constitué par un canal à écoulement libre de $19 \mathrm{~m}$ de largeur, disposé parallèlement aux conduites forcées. La dissipation d'une grande partie de l'énergie est obtenue sur le coursier du déchargeur grâce à la présence de 2000 《dents» de $0,60 \times 0,60 \mathrm{~m}$ de section et de 5 ou $15 \mathrm{~cm}$ de hauteur.

Après un service prolongé à la mise en service de l'usine ( 1 milliard de mètres cubes sont passés par le déchargeur), on a observé des érosions, localisées le plus souvent dans la partie amont du déchargeur. Les dégradations étaient de faible pro- fondeur sur les dents et se situaient généralement au niveau de leur enracinement sur le radier où le béton présentait une surface irrégulière et rugueuse et quelques vacuoles.

\section{Dégradations observées à l'aval de rainures de vannes.}

Nous avons déjà cité les érosions observées au barrage de Rivières, sur le Tarn. Cette manifestation de la cavitation se rencontre vraisemblablement lorsque la charge amont est suffisante pour provoquer une résorption brutale des vortex s'échappant des rainures des vannes; l'implosion des bulles d'air s'accompagne alors d'ondes de pression destructrices des bétons.

Il semble que les conditions favorables soient atteintes à partir d'une quinzaine de mètres de charge et sans doute également lorsque la section de la rainure se prête à la formation de tourbillons.

A Rivieres, la charge est de $17 \mathrm{~m}$ d'eau au niveau du seuil des vannes (fig. 4). Les rainures ont $2 \mathrm{~m}$ de lonsueur et $0,90 \mathrm{~m}$ de profondeur. Les zones dégradées affectent une forme triangulaire et sont situées immédiatement à l'aval des rainures. Leur hauteur est comprise entre 1 el $1,60 \mathrm{~m}$ et leur longueur entre 0,60 et $1 \mathrm{~m}$. Leur profondeur est, en général, comprise entre 6 et $10 \mathrm{~cm}$ (photos III et IV).

A Cize-Bolozon, sur l'Ain, la charge au niveau du seuil des vannes Stoney est très comparable $(16,50 \mathrm{~m})$. Les vannes sont à double corps. Il est possible d'évacuer $675 \mathrm{~m}^{3} / \mathrm{s}$ par la partie inférieure. Les rainures de vannes ont 2,23 $\mathrm{m}$ de longueur et $1 \mathrm{~m}$ de profondeur.

Après un fonctionnement de $400 \mathrm{~h}$ environ en «vanne de fond » et pour un débit moyen évacué de $400 \mathrm{~m}^{3} / \mathrm{s}$, tous les bajoyers des piles présentent une zone érodée de forme triangulaire, accolée aux rainures et ayant les dimensions approximatives suivantes : hauteur : 20 à $30 \mathrm{~cm}$, longueur : $50 \mathrm{~cm}$, profondeur : 10 à $25 \mathrm{~cm}$. Les érosions se sont produites dès la première année d'exploitation et ont fait l'objet de réparations périodiques.

Le même type d'érosion a été observé à Châteauneuf-du-Rhóne, à l'aval des rainures du batardeau amont du déchargeur (charge : $18 \mathrm{~m}$ ) et sur ladduction directe de la Rhue, dans la retenue de Bort-les-Orgues. Dans cette adduction, la galerie de $4 \mathrm{~m}$ de diamètre est fermée par une vanne à double étanchéité (dimensions : $3 \times 4 \mathrm{~m}$ ). La charge statique maximale sur la face amont de la vanne est de $39 \mathrm{~m}$. La contrepression due à la retenue est de $11 \mathrm{~m}$ au maximum. La vanne joue le ròle d'orifice de réglage et son ouverture, variable, est habituellement comprise entre 1 et $3 \mathrm{~m}$. L'adduction directe fonctionne d'une manière intermittente, au cours d'une période de deux mois environ chaque année.

Immédiatement en aval des rainures de la vanne, on a observé une zone cavitée sur chaque piédroit, d'une superficie de $1 \mathrm{~m}^{2}$ environ. Les érosions les plus importantes atteignaient la dimension du poing.

Un premier blindage de protection a été arraché. Un deuxième blindage, de $12 \mathrm{~mm}$ d'épaisseur, a été installé en janvier 1963 ; il est lié par soudure aux pieces fixes de la vanne. 


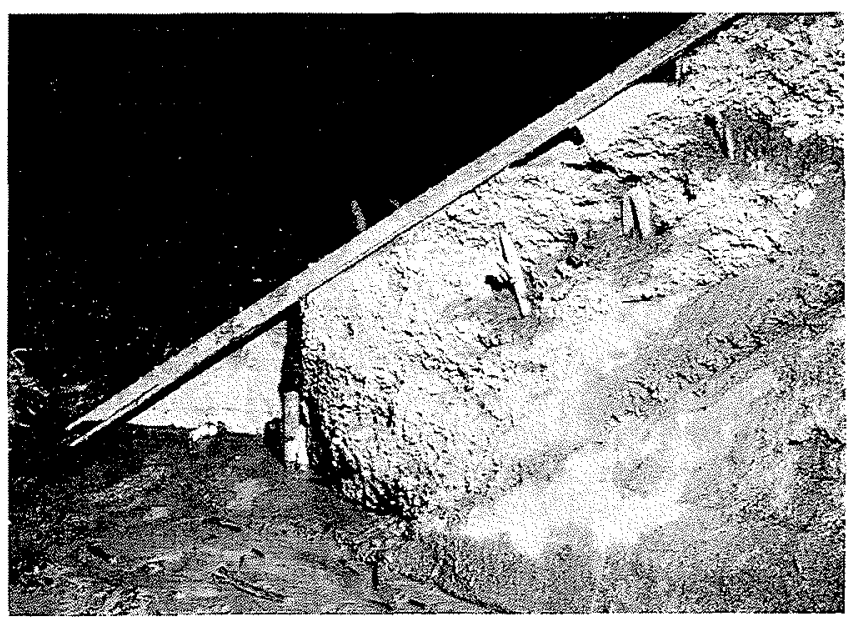

ENSEMBLE - GENERAL ARRANGEMENT

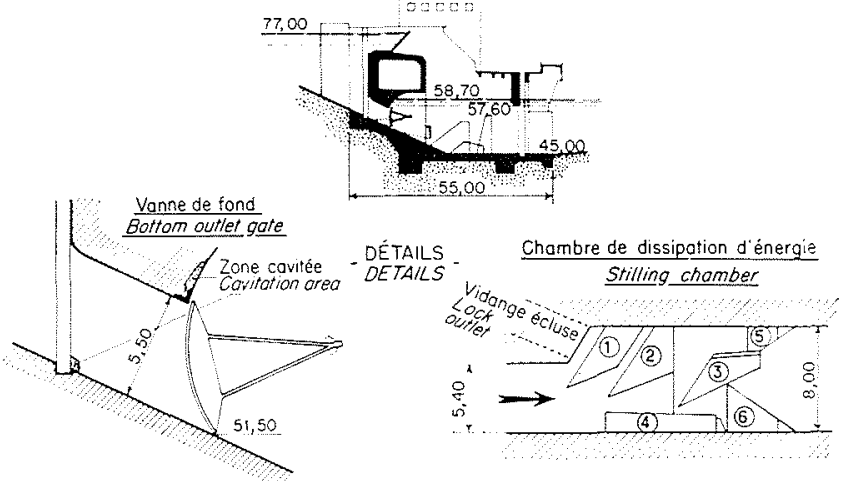

3/ Châteauneuf-du-Rhône: ensemble du déchargeur. Situation des dents de dissipation d'énergie.

Chateanneaf-du-Rhone: general arrangement of relief works. Energy dissipation teeth positions.

4/ Rivières:

ensemble d'un pertuis.

Rivières :

general arrangement

of a sluiceuray.

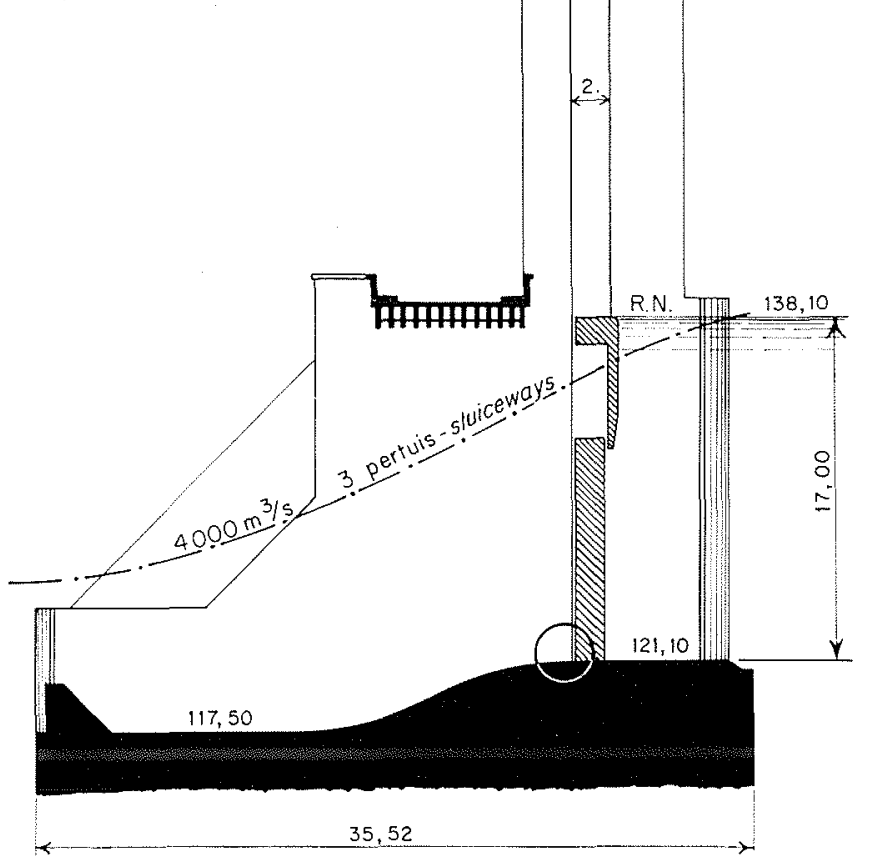

Photo II/ (Photopresse, Montitimar.) Cluâteauneuf'-clu-Rhòne:

Erosion de la dent $n^{*} 3$ protégée par un blindage de $10 \mathrm{~mm}$ (janvier 1964). Chat ealuneuf-du-Rhone: Erosion on $n^{\prime \prime} 3$ tooth, in spite of $10 \mathrm{~mm}$

thick plating protection (Iranuartl 1964).

(Photo E.D.F.) Photo Ill//

Rivières: Pertuis 3. Erosion du bajoyer rive droite. Rivieres: $n^{\prime \prime} 3$ sluicewa!l Right bank side wall erosion.
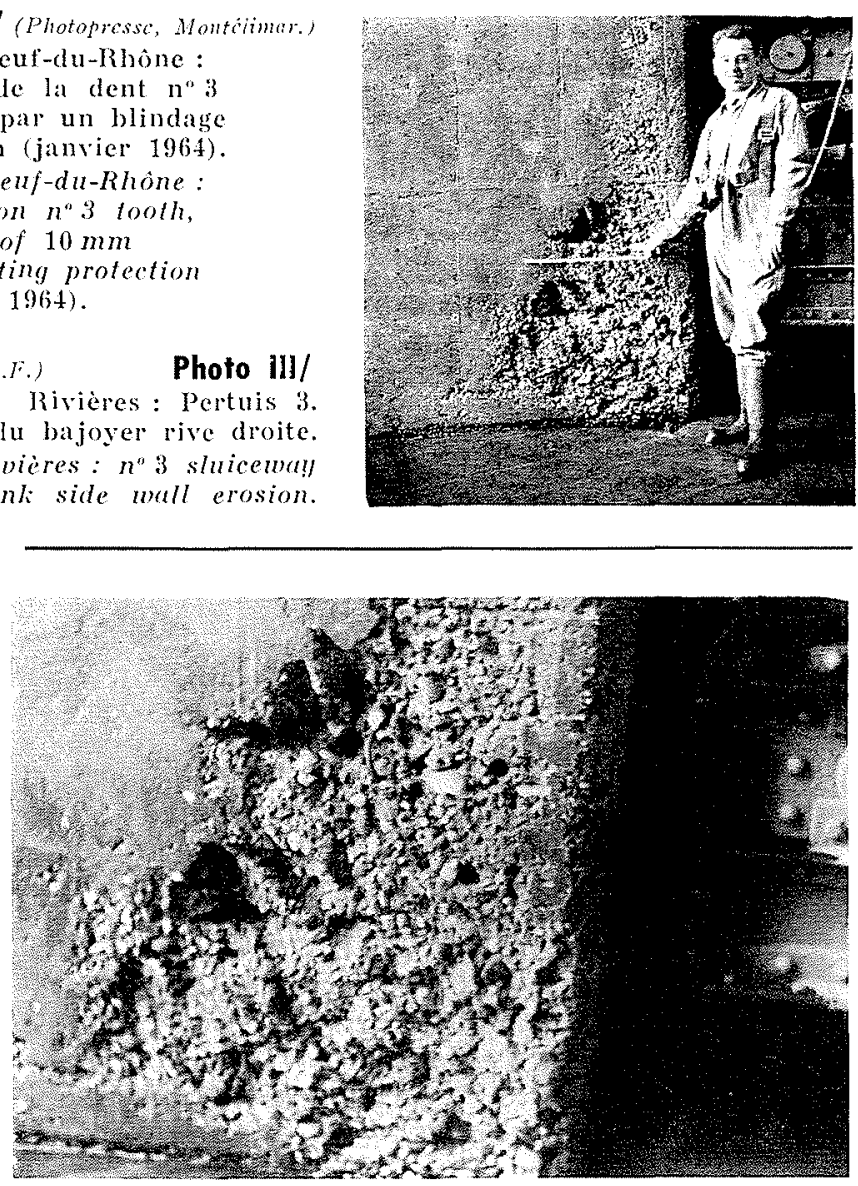

Photo IV/

(Photo E.D.F.) Rivières : pertuis 3. Erosion du bajoyer rive droite. Détails. Ribieres: $n^{n} 3$ sluicenaly. Right bank side wall erosion. Details.

VUE EN PLAN - PLAN VIEW

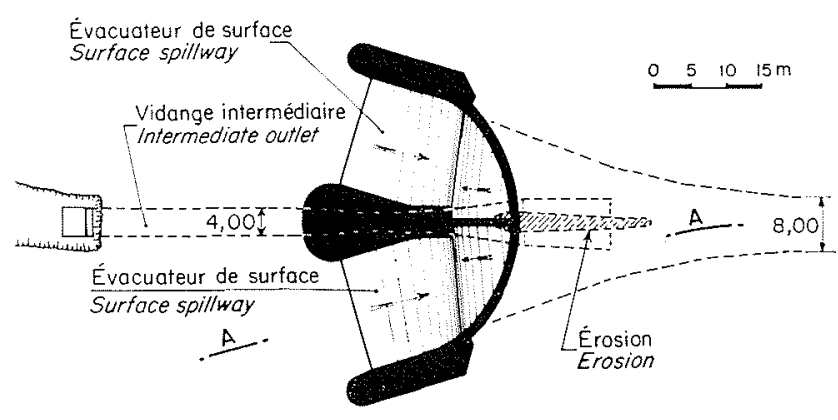

COUPE - SECTION A-A

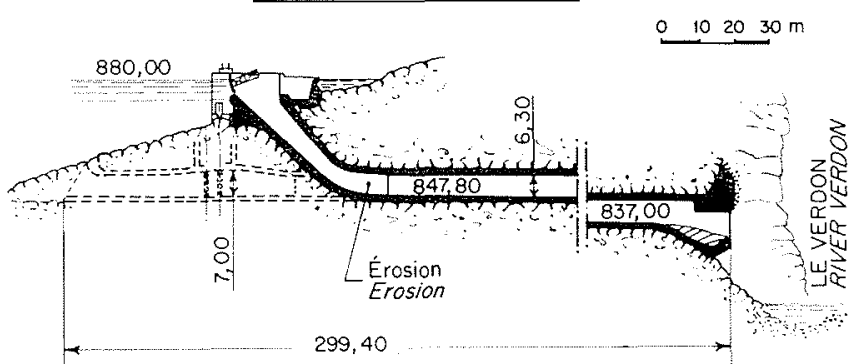

5/ Castillon : ensemble de l'évacuateur de surface et de la vidange intermédiaire.

Castillon: general arrangement of the surface spillway and intermediate outlet. 


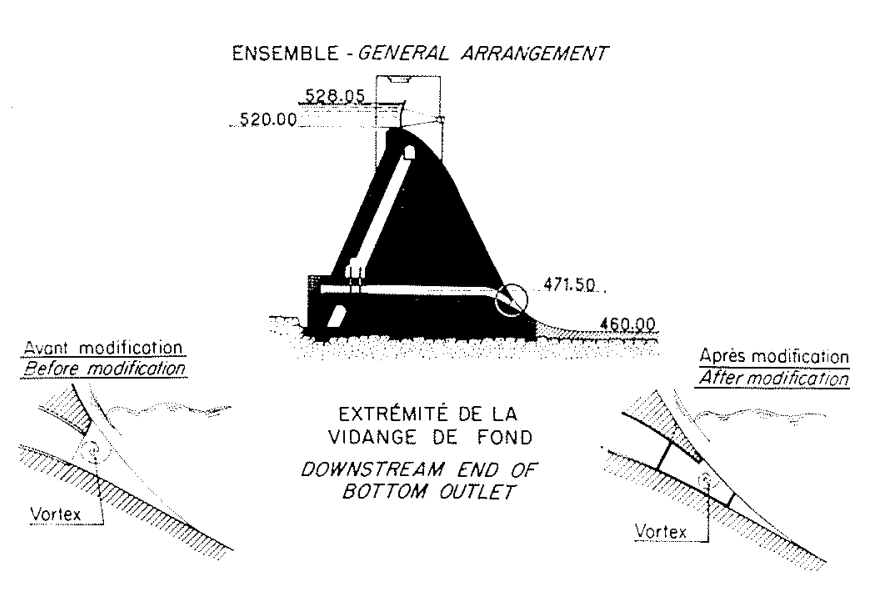

6/ Miranda : profil du barrage. Extrémité de la vidange de fond.

Miranda: general arrangement of the dam. Bottom ontlet details.

\section{Dégradations observées dans l'évacuateur de Castillon.}

Ces dégradations se situent au niveau du raccordement de la vidange intermédiaire avec le coursier de l'évacuateur de surface (fig. 5). La turbulence de l'écoulement, au droit du débouché de la vidange, est sans doute importante et c'est pourquoi nous avons rangé cet exemple d'érosion parmi les écolllements turbulents à vitesse moyenne modérée.

Le raccordement est situé $30 \mathrm{~m}$ au-dessous du niveau de la retenue normale. L'évacuateur de surface peut débiter, au total, $520 \mathrm{~m}^{3} / \mathrm{s}$ dans deux pertuis indépendants qui se raccordent au niveau du débouché de la vidange intermédiaire.

L'extrémité du mur, qui divise en deux pertuis l'évacuateur, partage également le débouché de la vidange en deux orifices. C'est sur la face rive droite de ce mur diviseur, dans la zone de raccordement des deux ouvrages, que l'on observe l'érosion du béton sous la forme de sept sillons.

Ils ont une longueur moyenne de $1 \mathrm{~m}$, une largeur de $20 \mathrm{~cm}$ et une profondeur maximale de $10 \mathrm{~cm}$. Ils sont situés exactement dans le prolongement de découpes du coffrage, sensiblement perpendiculaires à l'écoulement, et qui ont laissé subsister des balerres ou des décrochements de 10 à $25 \mathrm{~mm}$ de hauteur, encore apparents au-dessus des érosions (photos $\mathrm{V}$ et $\mathrm{VI}$ ).

Il est peut-être possible de rapprocher cel exemple des érosions observées au Portugal, sur les deux barrages de Bemposta et de Miranda (*) au débouche des vidanges de fond sur le parement aval de l'ouvrage qui sert de coursier à l'évacuateur de surface (fig. 6). Des essais sur modèle réduit ont montré, qu'avec la vidange fermée, le fonctionnement du déversoir s'accompagnnait, au débouché de la vidange, d'un vortex à axe horizontal. On a obtenu, tris approximativement, sur le modele, pour un débouché réalisé avec un mortier à faible teneur en liant, les zones d'érosion du prototype.

(*) Rapport 1-21 au XI" Congres A.I.R.H. (Leningrad $1955)$.

Rapport 12, question 33. IX Congres des Grands Barrages (septembre 1967 ).
Exemple de dégradations par cavitation au sein d'écoulements à vitesse moyenne élevée et à faible turbulence

\section{Evacuateurs de crues.}

On pouvait penser, a priori, que les ouvrages qui sont le siège d'écoulements à vitesse moyenne élevée (évacuateurs en saut de ski, vidanges de fond, etc.) permettraient l'observation d'un certain nombre d'érosions par cavitation. Ce n'est pas le cas, et nous citerons uniquement, dans celte rubrique, les importantes érosions observées dans la vidange de fond $n^{\circ} 1$ de Serre-Poncon. Il faut toutefois noter que la grande majorité des évacuateurs n'a pas connu, jusqu'à présent, une durée de fonctionnement aussi prolongée que la vidange de SerrePoncon. Citons quelques chiffres :

- à Chastang, les évacuateurs fonctionnent, en moyenne, chaque année, une vingtaine d'heures;

- à L'Aigle, entre $195 j$ et 1965 , les évacuateurs ont fonctionné, en moyenne, $180 \mathrm{~h}$ par an;

- à Bort-les-Orgues, de la mise en service (1951) à 1966, la durée cumulée de fonctionnement a été d'une centaine d'heures et les débits évacués compris entre 60 et $100 \mathrm{~m}^{3} / \mathrm{s}$.

Seul, l'évacuateur rive droite de Génissiat a eu un fonctionnement important, environ $24100 \mathrm{~h}$, depuis sa mise en service en avil 1948 .

Aucun de ces ourrages n'a présenté, à ce jour, de dégradations importantes et les quelques défectuosités des coursiers semblent, le plus souvent, devoir être attribuées à une mauvaise qualité des betons, au gel on à l'usure (photo VII).

\section{Vidange de fond $n^{\circ} 1$ de Serre-Ponçon.}

Chacune des deux vidanges de fond de SerrePoncon est équipée d'une vanme de garde et d'une vanne de réglage, distantes de $7 \mathrm{~m}$. La section d'écoulement au droit de la vanne de réglage a $5,20 \mathrm{~m}$ de hauteur et $2,60 \mathrm{~m}$ de largeur.

Le seuil de la vanne est calé à $124 \mathrm{~m}$ au-dessous du niveau de la retenue normale et les vitesses peuvent atteindre $45 \mathrm{~m} / \mathrm{s}$ (fig. 7 ).

A l'aval de la vanne de réglage, la section rectansulaire est raccordée progressivement, par des formes simples, sur $45 \mathrm{~m}$ de longueur à la section circulaire de $9,30 \mathrm{~m}$ de diametre. Le raccordement a été étudié sur modèle réduit. Un blindage en acier mi-dur, de $20 \mathrm{~mm}$ d'épaisseur, s'étend depuis $5 \mathrm{~m}$ à l'amont de la vanne de garde jusqu'à $15 \mathrm{~m}$ à l'aval de la vanne de réglage.

\section{Fonctionnement :}

Fin aoùt 1959 , la dérivation $\mathrm{n}^{\circ} 2$ a été fermée et la vidange $n^{\circ} 1$ mise en service. Cette dernière a fonctionne pratiquement sans interruption jus-

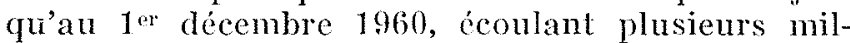
liards de mètres cubes d'eau. Au cours de cette période denx érosions importantes du radier ont été constatées. 


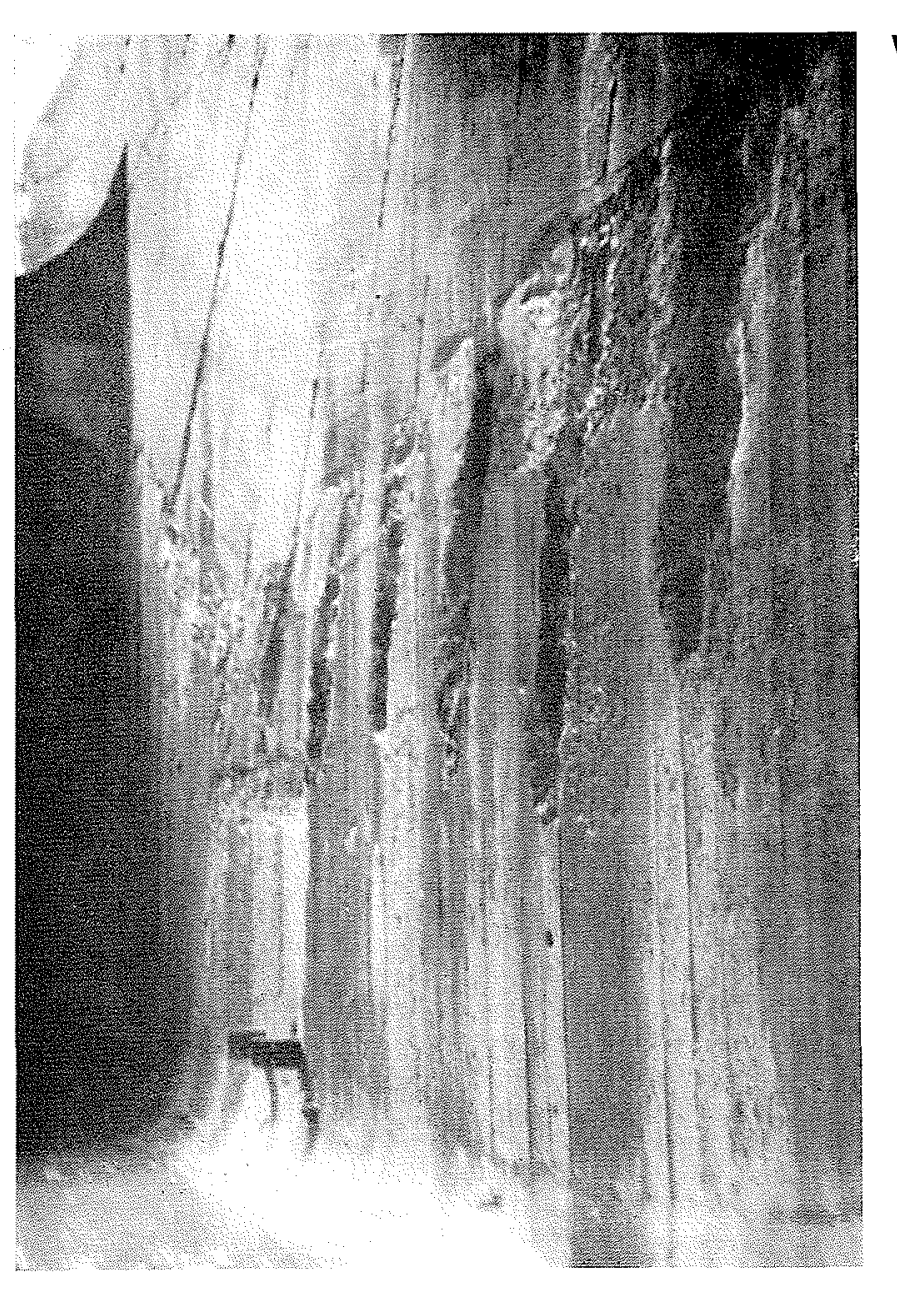

Photo V/

(Photo E.D.F.)

Castillon : évacuateur de crues. Erosions au raceordement de la vidange intermédiaire.

Castillon: Spillway erosion at the transition to the intermediate outlet.

Photo VII/

(Photo E.D.F.

Chastang : détail du coursier de

l'évacuatenr dans la zone médiane.

Chastang: ditails of central part of

spillual channel.

Serre-Ponçon : ensemble

de la vidange.

de fond $n^{\circ} 1$.

Détail de la

vanne de réglage.

Serre-Poncon :

general

arrangement of $n^{\circ} 1$ bottom outlet.

Control gate

details.

\section{7/ Élévation - Elevation}

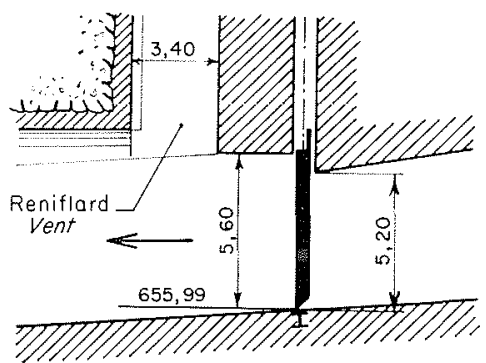

v

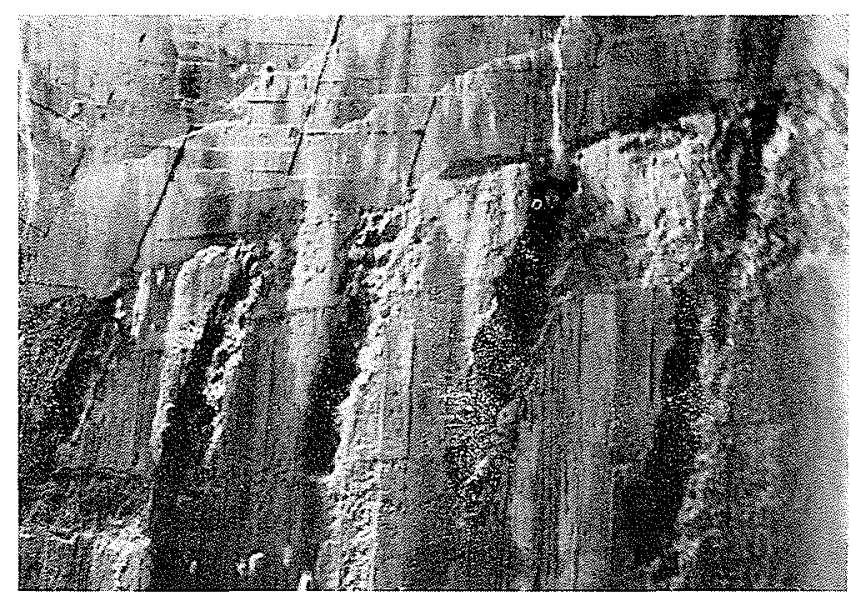

$1-$

n

e,

$1-$

et

s-

II

1

$\gg$,

la

it

le

le

1

ze

VII

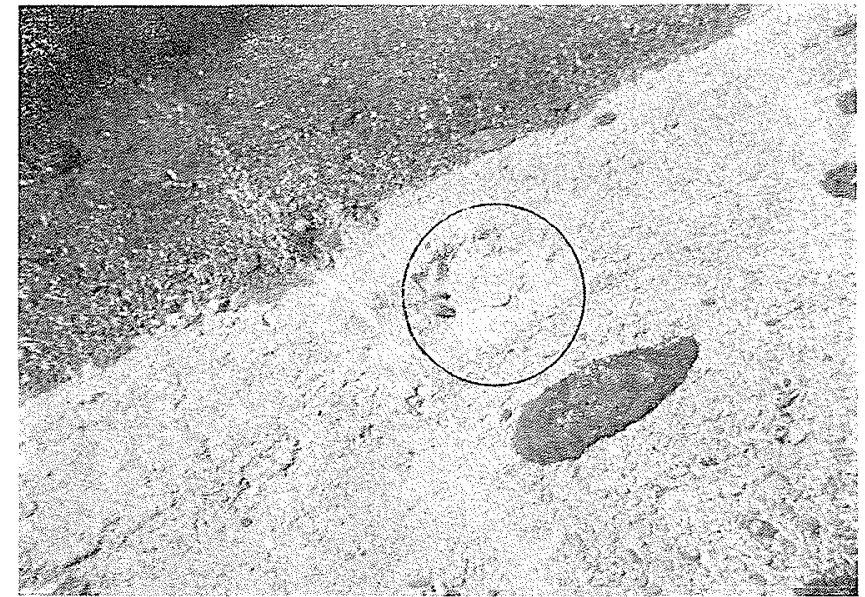

Photo VI/

(Photo R.D.F.)

Castillon : évacuateur de crues. Erosions au raccordement de la vidange intermédiaire. Détails. / Castillon: Spillwall erosion at the transition to the RN 780,00 intermediate ontlet. Details.

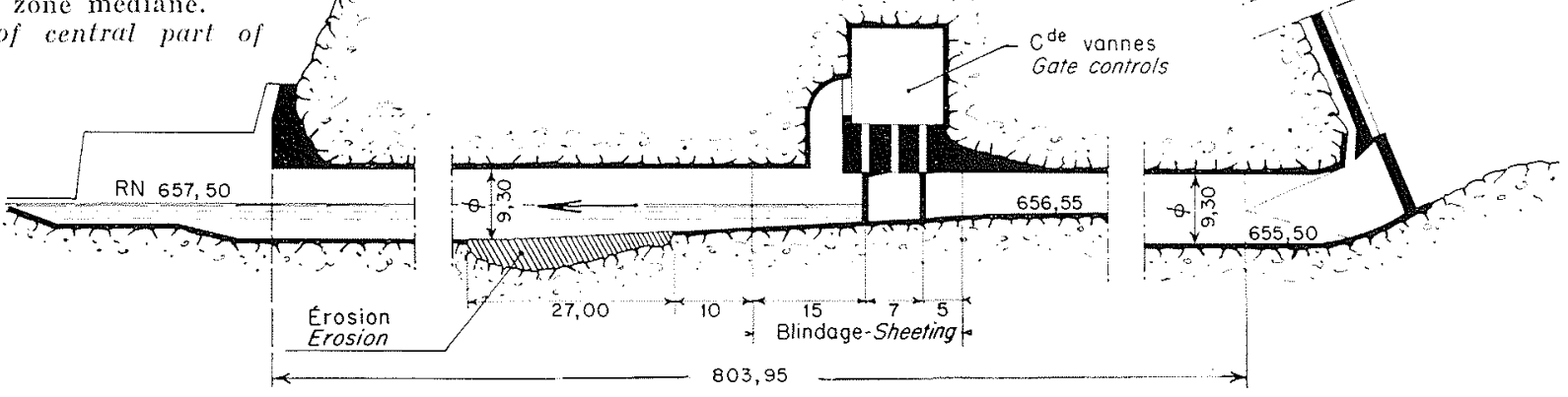

VANNE DE RÉGLAGE CONTROL GATE

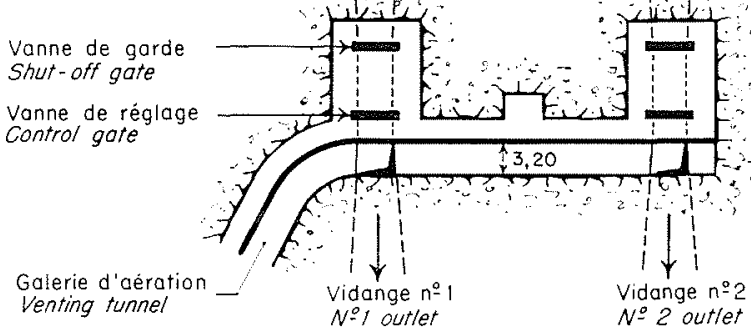


apporte un ensemble d'informations très interessant ef très précis, dont il faut louer les anteurs et plus genéralement, les services d'E.D.F. Les observations, bien entendu, ont porté non pas directement sur le phénomene hydraulique de cavitation sur l'ourage en service, mais seulement sur les erosions attribuees à la cavilation, et constatés après mise a sec de l'ouvage. Pour tous les exemples présentés, on se trouve en présence d'ecoulements très complexes, dont lat simulation sur modèle réduit, qui n'a d'alleurs pas eté tentéc, aurait été très delicate. La quasi-totalité des érosions constatées est liée a des perturbations locales : c'est le cas, par exemple, de la galerie de Serre-Ponçon, pour laquelle l'crosion semble liée a ressaut a l'aval de la vanue.

M. Drponer précise que l'un des buts de lenquête était dobtenir des informations sur les deteriorations pouvant survenil en l'absence de macroturbulence dans les ecoulements uniformes a très grande vitesse; les renseignements recueillis sur les évacuateurs de crue paraissent rassurants, mais ils ne permettent pas de dire si, dans un souci d'économie, il est possible d'augmenter encore les vitesses dans ces ourrages.

M. Caxayesis remarque que les déteriorations constatees dépendent de la qualité des bétons utilisés. 1-t-on, dans ce fomaine, des résultats d'essais effectues suivant des méthodes analogues à celles permettant de sélectionner les métaux resistant le mieux a la eavitation dans les turbomachines?

M. DUpont répond qu'un programme d'etude systématique de tenue des bétons en présence de grandes vitesses d'écoulement avait dé élaboré en liaison avec E.D.F.; il avait pour objectif de comparer les differents types de beton et surtout de formuler les tolérances acceptables pour l'irregularite de leur surface, compte tenu des conditions hydrodynamiques. Les installations d'essai sont d'une importance et d'un prix très supérieurs à leurs homologues utilisés pour les recherches sur la résistance des métaux à la cavitation, car pratiquement, il n'est pas possible de representer les bétons et mème les couches limites des écoulements a grande vitesse autrement qu'à l'échelle $1 / 1$.

Ce programme de recherche a été pour l'instant abandonne; on ne peut que souhaiter qu'il soit repris bientot.

Dans la littérature technique, on troure le compte rendu d'essais de cavitation effectués sur des eprouvettes en beton, en particulier, sous l'égide du Burean of Reclamation(?) des Etats-Unis; maheureusement, ces articles napportent que des réponses incompletes au probleme qui nous occupe.

M. LabAe précise que le programme d'essais, auquel a fait allusion M. Dupont, avait eté mis sur pied pour parfaire les spécifacations nécessaires a la réalisation d'un ouvage en projet dans les Alpes. Cet ouvrage ayant éte remplacé par un autre très différent, les essais n'ont pas été commandes.

II ajoute que de tels essais ont été réalisés non seulement aux U.S.A. mais aussi en U.R.S.S.; il ignore si ces derniers ont donné lieu a publication.

Il demande a M. Berxarn si, dans des cas d'erosions demandant des interventions d'entretien répétées: Cize Bolozon, par exemple, on a essayé des bétons de résine. La Rérion d'Equipement Alpes-Nord a essayé de tels bétons ou mortiers a l'abrasion sur l'Arveyron de la Mer de Glace, sans que les résultats soient vaiment conclaants.

M. Bernarb répond qu'une tentative d'emploi de tels bétons a été réalisce dernièrement a Rivières-sur-Tarn; il est trop tot pour conclure.

Le Président lève la séance en remerciant $M$. Destexay et M. Bernard pour leur communication qui a suscité des réactions de nature à encourager la poursuite du travail de la «ection Machines » dans ce domaine difficile.

\section{Abstract \\ Examples of cavitation damage to concrete \\ by J. Destenay * and J. Bernard * *}

In 1965 the Hydraulic Production Section of Electricite de France carried out a survey of cavitation damage to concrete among operational structures. Twenty cases were observed, resulting in the following findings:-

1) Cases of erosion were fairly infrequent;

2) Comparatively major damage usually occured after exceptionally prolonged operation (e.g. after passing part or the whole of the flow diverted through the relief arrangements during staggered starting-up of power sets;

3) More severe damage seems liable to occur with the two following types of flow:-

a) Moderate mean flow velocities $(10-20 \mathrm{~m} / \mathrm{sec})$ with considerable turbulence,

b) High mean flow velocities (above $30 \mathrm{~m} / \mathrm{sec}$ ) with little turbulence.

Relief works belong to category $(a)$. Their energy dissipation arrangements frequently seem to require full sheeting protection.

Where high flow velocities are experienced, e.g. in bottom outlets, careful surface outline design and a smooth finish are important factors in preventing rapidly spreading damage.

- Ingénieur-Conseil auprès d'E.D.F.

* Ingénieur au Service de la Production Hydraulique d'E.D.F. 


\section{Erosion constatée en juin 1960:}

Après un fonctionnement avec des débits compris entre 155 et $415 \mathrm{~m}^{3} / \mathrm{s}$ (de la mi-mai à la fin juin) et pour une charge de $85 \mathrm{~m}$ environ, des bruits anormaux et violents ont conduit à visiter la galerie.

On a constaté la présence d'une fosse d'érosion qui débutait, à environ $10 \mathrm{~m}$ en aval du blindage, avee une profondeur de $40 \mathrm{~cm}$ (au droit d'un changement de pente de moins de 1 degré du radier) et s'étendait sur $27 \mathrm{~m}$ de longueur. Elle s'approfondissait progressivement pour atteindre une profondeur maximale de $4 \mathrm{~m}$, sensiblement au droit du raccordement avec la section circulaire (en ce point, la pente du radier diminue avee un angle inférieur $\dot{a} 4^{\circ}$ ). Les surfaces érodées du béton, très irrégulicres, avaient un aspect très rugueux, « arraché », caractéristique de la cavitation. Le volume de la fosse était de $360 \mathrm{~m}^{3}$ environ.

Aucune trace appréciable de cavitation n'était visible sur les blindages sauf à l'aval immédiat de quelques irrégularités provenant de goultes de mortier qui n'avaient pas été nettoyées.

Les réparations furent effectuées rapidement avec du ciment HRI et la vidange remise en service le 7 juillet 1960 .

\section{Erosion constatée en septembre 1960:}

La vidange fonctionna, jusqu'en septembre, avec des débits inférieurs à $200 \mathrm{~m}^{3} / \mathrm{s}$ pour une charge d'environ $105 \mathrm{~m}$. Dans la deuxième quinzaine de septembre, il fut nécessaire d'évacuer $300 \mathrm{~m}^{3} / \mathrm{s}$ environ. Le passage de ce débit s'effectua normalement pendant une journée environ, après quoi, des bruits anormaux ayant commencé à se faire entendre et des à-coups ayant été constatés dans le fonctionnement, la vidange fut arrêtée.

Sans vider la galerie, des sondages permirent de constater la présence d'une nouvelle fosse d'érosion, moins importante qu'en juin, et située plus en amont (photos VIII et IX). Le blindage en aval immédiat des rainures de la vanne de réglage était cavité sur une zone triangulaire de $1 \mathrm{~m}$ de haut sur $1 \mathrm{~m}$ de longueur environ; il était même percé en rive droite sur 6 dm² (photo $\mathrm{X}$ ). Les impératifs de la météorologic imposèrent une remise en service arec des débits compris entre 100 et $150 \mathrm{~m}^{3} / \mathrm{s}$. Un mois plus tard, aprés vidange de la galerie, la fosse d'érosion ne semblait pas avoir évolué de façon notable. Elle s'amorcait à $30 \mathrm{~cm}$ en aval du blindage par trois saignées qui s'approfondissaient et se rejoignaient pour former, à $10 \mathrm{~m}$ du blindage, un trou occupant toute la largeur de la galerie et atteignant le rocher avec une profondeur maximale d'environ $2 \mathrm{~m}$.

Le volume de la fosse étail de $150 \mathrm{~m}^{\circ}$. En de nombreux points, de petites irrégularités de surface de l'ordre de quelques millimetres avaient provoqué des amorces de cavitation en aval immédiat.

La réparation définitive a consisté à réaliser un revètement en béton d'un état de surface au moins équivalent à celui d'un blindage métallique par réglage soigné et meulage de toute la surface.

Des essais ultérieurs, au débit maximal de $600 \mathrm{~m}^{3} / \mathrm{s}$, mais de faible durée (5 h $30 \mathrm{~mm}$ ), n'ont montré aucune érosion appréciable.

$$
\text { ה: }
$$

Ces exemples montrent quelques manifestations de la cavitation dans les ouvrages hydroélectriques. Celles-ci semblent rares et, le plus souvent, de faible ampleur pour les ouvages actuellement en service.

Pour les écoulements à vitesse modérée (10 à $20 \mathrm{~m} / \mathrm{s}$ ), mais à forte turbulence, il semble possible de se mettre à l'abri de la plupart des dégradations, gràce à certaines précautions : blindage des zones de dissipation d'énergie dans les déchargeurs et blindage des bajoyers, à partir d'une certaine charge, à l'aval des rainures de vannes ou de batardeaux.

Pour les écoulements à grande vitesse $(40 \mathrm{~m} / \mathrm{s})$, il semble nécessaire de veiller très soigneusement aux formes et aux états de surface, les dégradations pouvant s'amplifier rapidement.
Avant de donner la parole à M. Benvand pour l'expose de la communication ci-dessus, $M$. le Président présente celle-ei dans les termes suivants:

"Le titre propose initialement par la « Section Machines $\gg$ pour ee travail, était :

« Recensoment des cas oì ont été observées des cavilations sur les ouvrages de génie civil. 》

Ce recensement était mené par un groupe dont avait hien voulu se eliarger M. DEstevat.

M. Bennard, a l'instigation de M. Destenay, faisait en 1965 une importante enquete sur le sujet.

En principe, ce travail devait etre ensuite examiné et discuté par un groupe de la « Section Machines 》 pour essaper d'en tirer des observations et si possible des conclusions. Malheureusement, cette derniere partie du travail n'a pas encore éte faite et e'est pourquoi le rapport qui vous est présenté aujourd'hui porte le titre :

"Quelques exemples de dégradation des bétons par cavifation dans des ouvrages hydrólectriques. »
Je tiens à remercier M. Pousse d'avoir bien roulu acepter que ses services présentent ce rapport sous sa formc actuelle. D'une part, le recensement des résultats constatés apporte des renseignements très précieux et d'autre part, il ne peut que stimuler tous ceux gui s'intéressent à la cavitation et les inciter à se pencher d'une maniere plus efficace sur le mécanisme des érosions dans les ouvages de génie civil (par exemple, en procédant à des essais systématiques en laboratoire afin de les comparer aux observations faites en grandeur nature) $\gg$.

Aprè lexposé de M. BErnard, M. le président remercie ce demier et ouvre la discussion.

M. Canaveris demande si l'on a pu meltre en évidence une relation entre certains risques d'érosion et certaines formes de cavitation au moins dans quelques cas simples et bien définis : cavitation derrière un tube cylindrique, à l'a val d'un redans à talus à $30^{\circ}$, etc.

M. Durone confirme que Ja communication de M. Bernaris 
apporte un ensemble d'informations très intéressant et très précis, dont il faut louer les auteurs et plus généralement, les services d'E.D.F. Les observations, bien entendu, ont porté non pas directement sur le phénomène hydraulique de cavitation sur l'ourrage en service, mais seulement sup les érosions attribuées a la cavitation, et constatées après mise a see de l'ouvage. Pour tous les exemples présentés, on se trouve en presence d'ecoulements tres complexes, dont la simulation sur modele réduit, gui n'a d'ailleurs pas été tentée, aurait été trés delicate. La quasi-totalité des érosions constatées est liee a des perturbations locales : c'est le cas, par exemple, de la galerie de Serre-Ponçon, pour laquelle l'érosion semble lice au ressaut a l'aval de la vanne.

M. Drrore précise que l'un des buts de l'enquête étail dobtenir des informations sur les détériorations pouvant survenir en l'absence de macroturbulence dans les écoulements uniformes a très grande vitesse; les renseignements recueillis sur les evacuateurs de crue paraissent rassurants, mais jls ne permettent pas de dire si, dans un souci d'économie, il est possible d'augmenter encore les vitesses dans ces ouvrages.

M. Caxavels remarque que les déteriolations constatés dépendent de la qualité des bétons utilisés. A-t-on, dans ce domaine, des résultats d'essais effectués suivant des méthodes analogues à celles permettant de sélectionner les métaux résistant le mieux a la caritation dans les turbomachines?

M. Duport répond qu'un programme d'etude systématique de tenue des bétons en présence de grandes vitesses d'écoulement avait eté blaboré en liaison avec E.D.F.; il avait pour objectif de comparer les différents types de béton et surtout de formuler les tolémances acceptables pour l'irregularité de leur surface, compte tenu des conditions hydrodynamiques. Les installations d'essai sont d'une importance et d'un prix trés superieurs a leurs homologues utilisés pour les recherches sur la résistance des metaux a la cavitation, car pratiquement, il n'est pas possible de représenter les betons et mome les couches limites des écoulements a srande vitesse autrement qu'à l'échelle $1 / 1$.

Ce programme de recherche a eté pour l'instant abandonne; on ne peut que souhaiter qu'il soit repris bientot.

Dans la litterature technique, on trouve le compte rendu d'essais de cavitation effectués sur des éprouvettes en béton, en particulier, sous l'égide du Bureau of Reclamation (?) des Etats-Unis; malheureusement, ces articles n'apportent que des réponses incomplètes au probleme qui nous occupe.

M. LABAtE précise que le plogramme d'essais, auguel a fait allusion M. Drport, avait ete mis sur pied pour parfaire les spécifications necessaires a la réalisation d'un ouvrage en projet dans les Alpes. Cet ouvage ayant été remplacé par un autre très différent, les essais n’ont pas été commandés.

Il ajonte que de tels essais ont eté réalisés non senlement aux U.S.A. mais aussi en U.R.S.S.; il ignore si ces derniers ont donné lieu à publication.

Il demande à M. Benxaro si, dans des cas d'erosions demandant des interventions d'entretien répétées: Cize Bolozon, par exemple, on a essayé des bétons de résine. La Région d'Equipement Alpes-Nord a essayé de tels bétons ou mortiers a l'abrasion sur l'Arveyron de la Mer de Glace, sans que les résultats soient vaiment concluants.

M. BEnnand répond qu'une tentative d'emploi de tels bétons a été réalisće dernièrement à Rivières-sur-Tarn; il est trop tôt pour conclure.

Le Président jève la séance en remerciant $M$. Destexar et M. BERNaro pour leur communication qui a suscité des réactions de nature à encourager la poursuite du travail de la «Section Machines » dans ce domaine difficile.

\section{Abstract \\ Examples of cavitation damage to concrete}

by J. Destenay * and J. Bernard * *

In 1965 the Hydraulic Production Section of Electricite de France carried out a survey of cavitation damage to concrete among operational structures. Twenty cases were observed, resulting in the following findings:-

1) Cases of erosion were fairly infrequent;

2) Comparatively major damage usually occured after exceptionally prolonged operation (e.g. after passing part or the whole of the flow diverted through the relief arrangements during staggered starting-up of power sets;

3) More severe damage seems liable to occur with the two following types of flow:-

a) Moderate mean flow velocities $(10-20 \mathrm{~m} / \mathrm{sec})$ with considerable turbulence,

b) High mean flow velocities (above $30 \mathrm{~m} / \mathrm{sec}$ ) with little turbulence.

Relief works belong to category $(a)$. Their energy dissipation arrangements frequently seem to require full sheeting protection.

Where high flow velocities are experienced, e.g. in bottom outlets, careful surface outline design and a smooth finish are important factors in preventing rapidly spreading damage.

- Ingénieur-Conseil auprès d'E.D.F.

* Ingénieur au Service de la Production Hydraulique d’E.D.F. 